\section{Cracking the BAFF code}

Fabienne Mackay ${ }^{*}$ and Pascal Schneider

* The Autoimmunity Research Unit, the Garvan Institute of Medical Research, 384 Victoria Street, Darlinghurst, NSW 2030, Australia

Department of Biochemistry, University of Lausanne, Chemin des Boveresses 155, Epalinges, CH-1066, Switzerland

Corresponding author: Professor Fabienne Mackay, $\mathrm{PhD}$

The Autoimmunity Research Unit, the Garvan Institute of Medical Research, 384 Victoria Street, Darlinghurst, NSW 2030, Australia.

Tel: +61 292958414

Fax: +61 292958404

Email: f.mackay@garvan.org.au
Abstract:

B-cell activating factor from the TNF family (BAFF) and APRIL are crucial survival factors for peripheral B cells. An excess of BAFF leads to the development of autoimmune disorders in animal models, and high levels of BAFF have been detected in the serum of patients with various autoimmune conditions. We discuss that in mice, autoimmunity induced by BAFF seems to be linked to $\mathrm{T}$ cell-independent $\mathrm{B}$ cell activation rather than to a severe breakdown of B-cell tolerance. We also outline the mechanisms of BAFF signalling, the impact of ligand oligomerization on receptor activation, and the progress of BAFF-depleting agents in the clinic. 
Immune cells produced in the bone marrow undergo a process of quality control, known as immune tolerance, that occurs throughout the life of B and T cells and that carefully selects useful protective immune cells while eliminating or neutralizing potentially harmful self-reactive cells ${ }^{1}$. The elimination or reprogramming of selfreactive $\mathrm{B}$ cells through $\mathrm{B}$-cell receptor $(\mathrm{BCR})$ editing $[\mathbf{G}]$ takes place during $\mathrm{B}-\mathrm{cell}$ development in the bone marrow, during B-cell maturation in the spleen and most probably after activation, following affinity maturation in a germinal centre ${ }^{2}$.

The nature of BCR engagement ${ }^{3}$ and the availability of survival cytokines, such as interleukin-7 and B-cell activating factor from the TNF family (BAFF), are key determinants deciding the life or death of developing B cells during the establishment of tolerance ${ }^{1}$. Thus, the balance between antigen receptor-driven stimuli and signals from survival cytokines controls the quality of most emerging immune cells. It was therefore tempting to assume that the autoimmune symptoms that are observed in BAFF over-expressing mice are due to the rescue of auto-reactive B cells from deletion. This Review describes that this is unlikely to be the only possible explanation for the association between BAFF and autoimmunity; new evidence implicates BAFF in the pathogenic activation of T cell-independent, lowaffinity self-reactive B cells. At a time when BAFF antagonists are being actively tested in clinical trials for autoimmunity and lymphomas, this Review also summarizes our knowledge of how BAFF and its closely related homologue APRIL (a proliferation-inducing ligand) activate their receptors and transmit survival and growth signals to B cells.

\section{Ligands in the BAFF system}

The tumour-necrosis factor (TNF)-family ligands BAFF (also known as TNFSF13B, BLyS or TALL-1) and APRIL (also known as TNFSF13) are homotrimeric type II transmembrane proteins. A membrane-bound form of APRIL, TWE-PRIL, is generated by trans-splicing [G] of the adjacent TWEAK (TNF-related weak inducer of apoptosis) and APRIL genes ${ }^{4}$, but little is known about the physiological function of TWE-PRIL.

BAFF and APRIL are proteolytically processed at furin consensus sequences to produce soluble cytokines (FIG 1). The processing of membrane-bound APRIL is very efficient ${ }^{4}$, explaining why epitopes located before and after the processing site fail to co-localize by immunohistochemistry ${ }^{5,6}$. BAFF, however, can also be found in a membrane-bound form. In myeloid cells, BAFF processing is increased in a Fc!RI-dependent manner by the binding of immune complexes ${ }^{7}$. Processed, soluble BAFF adopts the usual trimeric form of a TNF-family ligand, but it is the only member of the family that can also further assemble as an ordered, capsid-like structure comprising twenty trimers. The receptor-binding sites remain exposed and accessible at the surface of BAFF 60-mers, as shown by crystallography ${ }^{8}$. Heteromers of BAFF and APRIL have also been described ${ }^{4}$.

\section{Receptors in the BAFF system}

BAFF and APRIL both bind to the receptors B-cell maturation antigen (BCMA; also known as TNFRSF17) and transmembrane activator and calcium-modulator and cyclophilin ligand interactor (TACI; also known as TNFRSF13B), and BAFF additionally binds to BAFF receptor (BAFFR; also known as BR3 or TNFRSF13C) ${ }^{4}$

9. Systematic mutagenesis studies of the relevant portions of the relatively small receptors TACI, BCMA and BAFFR have identified essential determinants of ligand binding as well as residues that account for the lack of interaction between APRIL and BAFFR, and for the weak binding of BAFF to BCMA ${ }^{10}$ (reviewed in ${ }^{4,9}$ ). In addition to TACI and BCMA, APRIL also interacts with the polysaccharide side chains of heparan sulphate proteoglycans (HSPGs) (FIG 1A). This interaction is mediated by basic residues of APRIL and does not interfere with binding to either TACI or BCMA ${ }^{11,12}$. It is noteworthy that HSPG-bound APRIL is biologically active 5,13

TACI exists as two splice variants containing a high-affinity ligand-binding site preceded or not by a low-affinity binding site of unknown relevance ${ }^{10}$. TACI, similarly to APRIL, interacts with proteoglycans such as syndecans ${ }^{14}$, which are expressed on the surface of many mesenchymal cells but also on activated macrophages and antibody-secreting cells. Syndecans might function as local activators of APRIL ${ }^{5,13}$, or as ligands that activate signalling through TACI ${ }^{14}$, or as direct providers of signals to antibody-secreting cells ${ }^{15}$.

Until recently, it was questionable whether the low-affinity binding of BAFF to BCMA was physiologically relevant. However, BCMA-dependent plasma cells in the bone marrow disappear if both APRIL and BAFF are removed by administration of TACI-Fc, but the number of these cells is not affected by selective removal of 
either BAFF alone (by administration of BAFFR-Fc) or APRIL alone (in April ${ }^{/-}$ mice) ${ }^{16}$, which indicates that BCMA can deliver relevant signals in response to both BAFF and APRIL in vivo. The weak BAFF-BCMA interaction is most probably stabilized by avidity effects ${ }^{17}$.

\section{Signal transduction by ligand oligomerization}

A trimeric ligand such as BAFF or APRIL binds to three independent receptors to initiate signalling ${ }^{8,10}$. Because more than one receptor is required to transmit the signal, the system is susceptible to dominant effects of mutations, as is probably the case in patients with common variable immunodeficiency (CVID) harbouring heterozygous TACI mutations (Box 1) ${ }^{18,19}$.

Binding of a trimeric ligand to its cognate receptor does not, however, necessarily result in productive signalling. This is particularly apparent for TACIdependent signals in response to APRIL stimulation in primary B cells. A single trimer of APRIL binds to TACI, but APRIL is essentially inactive unless it is further multimerized, for example by HSPG ${ }^{12,13}$. Similarly, BAFF 60-mer but not BAFF 3mer signals through TACI, although both forms of BAFF can initiate BAFFRmediated effects ${ }^{20}$. These in vitro data show that signalling through TACI requires oligomeric ligands such as BAFF 60-mer, HSPG-bound APRIL or membrane-bound ligands, but not soluble, trimeric ligands. Similar conclusions were reached for TACI expressed by monocytes and dendritic cells ${ }^{21,}{ }^{22}$. The observed requirement for oligomeric ligands to induce signalling through TACI fits with a model in which intracellular signalling molecules are recruited on adjacent ligand-receptor complexes (FIG 1B). TNF-receptor-associated factors (TRAFs) are trimeric intracellular proteins that bind linear TRAF-binding sequences present in several receptors, including BCMA, TACI and BAFFR. The affinity of TRAFs for a monomeric receptor is very low, but the interaction is greatly stabilized by avidity effects when the TRAF trimer binds to three receptors held in the correct geometry by the ligand ${ }^{23}$. TRAF2 and TRAF6 bind to TACI and potently activate the transcription factor nuclear factor- B (NF- B) if at least two trimeric TRAF molecules are held in close proximity ${ }^{24}$, a situation that can be achieved when at least six receptors are recruited (FIG 1B). The situation is probably different for BAFFR, which selectively recruits TRAF3 for the purpose of degrading it, shortcutting the need for higher order receptor clustering to obtain an effect ${ }^{25-27}$ (FIG 1B).

The early steps of BAFFR signalling have long remained elusive, because BAFFR binds only TRAF3, which by itself does not activate the classical [G] or alternative NF- B pathways [G]. TRAF3 interacts with NF- B-inducing kinase (NIK), the upstream kinase responsible for NF- B2 processing, and induces NIK degradation ${ }^{28}$, resulting in decreased NF- B2 activation. Targeted deletion of TRAF3 (and/or TRAF2) in B cells leads to constitutive activation of NF- B2, recapitulating the effect of constant activation of BAFFR ${ }^{25}$. Although TRAF2 does not interact directly with BAFFR, its deletion induces TRAF3 accumulation ${ }^{25}$. These observations form the basis for a model that rationalizes the early steps of BAFFR signalling: in the absence of BAFF, TRAF3 binds to NIK and, with the help of TRAF2, induces NIK degradation by the proteasome, thus preventing activation of the alternative NF- B pathway. In the presence of BAFF, TRAF3 is recruited to BAFFR and degraded in a TRAF2-dependent manner, resulting in the stabilization of NIK and activation of NF- B2, which then leads to increased survival (FIG 2) ${ }^{29,30}$

In summary, signalling in the BAFF system is determined not only by the specificity of BAFF and APRIL for their respective receptors, but also by the form in which these ligands are presented to receptors.

\section{Expression of BAFF, APRIL and their receptors}

BAFF and APRIL are mainly produced by innate immune cells such as neutrophils, macrophages, monocytes, dendritic cells (DCs) and follicular dendritic cells (FDCs) (reviewed in ${ }^{31}$ ), and their expression is increased by type I interferon (IFN), IFN-!, IL-10 and granulocyte colony-stimulating factor (G-CSF) ${ }^{31,32}$, as well as by the activation of Toll-like receptors (TLRs) such as TLR4 ${ }^{31}$ or TLR9 ${ }^{33}$. T cells ${ }^{34}$, activated B cells ${ }^{35}$ and B-cell chronic lymphocytic leukaemia (B-CLL) cells can also produce BAFF and APRIL, contributing to tumour-cell survival in the case of B-CLL 36

A recent development in the field is the identification of non-haematopoietic cells expressing BAFF and/or APRIL. These include cytotrophoblasts [G] in the placenta ${ }^{37}$, fibroblast-like synoviocytes in the synovium of patients with rheumatoid 
arthritis ${ }^{38}$, osteoclasts in patients with multiple myeloma ${ }^{39,}{ }^{40}$, epithelial cells in tonsils ${ }^{5}$ and airways ${ }^{41}$, salivary gland epithelial cells in patients with Sjögren's syndrome ${ }^{42}$, breast adipocytes ${ }^{43}$, carcinoma cells ${ }^{43}$ and astrocytes in patients with primary central nervous system lymphomas or multiple sclerosis ${ }^{44,45}$.

In conclusion, activated innate immune cells and, to a lesser extent lymphocytes, produce BAFF and APRIL to support ongoing immune responses. Production of these ligands by non-haematopoietic cells might also provide local niches to modulate the survival and function of B cells and plasma cells in health and disease.

The receptors for BAFF and APRIL are mainly expressed by B cells ${ }^{46,47}$. In mice, BAFFR expression is low on newly formed immature B cells, but increases as these cells progress through the stages of B cell maturation, and is subsequently expressed by all mature B cells ${ }^{46}$. TACI is expressed by all peripheral B cells and particularly marginal-zone $[\mathbf{G}]$ and B1 B cells $[\mathbf{G}]$ (reviewed in ${ }^{48}$ ). BCMA expression is restricted to antibody-producing cells ${ }^{49}$. In humans, BAFFR is widely expressed on B cells, except bone-marrow plasma cells ${ }^{46,47}$. TACI is expressed by $\mathrm{CD} 27^{+}$memory B cells, tonsillar and bone-marrow plasma cells, a subpopulation of activated $\mathrm{CD} 27^{-}$, non-germinal-centre cells ${ }^{46,47,50}$ and a small subset of naïve $\mathrm{B}$ cells in the blood and tonsils ${ }^{46}$, which is consistent with the idea that, similar to the mouse system, TACI is an inducible receptor in humans. BCMA is expressed by plasma cells from tonsils, spleen and bone marrow ${ }^{46}$, but also by tonsillar memory B cells and by germinal-centre B cells, the latter being TACIBAFFR ${ }^{\text {low }} 47$.

Regarding non-B cells, BAFFR expression is upregulated on $\mathrm{T}$ cells after activation ${ }^{34}$ and it is constitutively expressed by regulatory $\mathrm{T}$ cells ${ }^{34}$, whereas TACI is expressed by monocytes ${ }^{21}$ and DCs ${ }^{22}$. These results indicate that the role of BAFF and APRIL extends beyond that of B-cell biology, although so far little is known of the potential effects.

\section{The phenotype of mice deficient for BAFF, APRIL or their receptors}

In the periphery, and possibly in the bone marrow, newly formed immature B cells differentiate to immature transitional type 1 (T1), type 2 (T2) and/or type 3 (T3) cells before becoming mature follicular or marginal-zone $\mathrm{B}$ cells ${ }^{51}$ (FIG 3). The nature of the BCR decides whether a B cell becomes follicular or marginal-zone, a decision that is taken at the $\mathrm{T} 2$ stage $^{52}$, or even as early as the $\mathrm{T} 1$ stage $^{53}$ (FIG 3). A survival role for BAFF and BAFFR during B-cell maturation was shown in BAFF- and BAFFRdeficient animals, in which B-cell maturation is impaired beyond the T1 stage ${ }^{31}$ (Table 1). However, B1 B cells, memory B cells and a small population of mature splenic B cells do not require BAFF or APRIL for survival ${ }^{16,31,54-56}$ (FIG 3). B-cell maturation in $\mathrm{April}^{/-}$mice is normal (reviewed in ${ }^{4}$ ), but class switching [G] to IgA in two independent $\mathrm{April}^{/-}$mouse strains is impaired ${ }^{4,57}$. APRIL is also important for the survival of class-switched mouse B cells ${ }^{58}$. APRIL and BAFF mediate CD40independent class switching to IgA through both TACI and BAFFR ${ }^{59,60}$, and binding of APRIL to HSPGs might be crucial in this respect because treatment of B cells with heparinitase $[\mathbf{G}]$ abrogated the class-switching response to APRIL ${ }^{15}$. In humans, intestinal epithelial cells produce APRIL in response to TLR activation, which locally drives TACI-mediated class switching from IgA1 to IgA2 ${ }^{61}$. These cells also produce thymic stromal lymphopoietin (TSLP) that is sensed by DCs in the lamina propria, which then release APRIL ${ }^{61,}{ }^{62}$. In the bone marrow, APRIL favours the establishment and/or the survival of plasmablasts, and lack of APRIL expression in the bone marrow of newborn mice might explain why antibody responses in neonates are short-lived ${ }^{58}$. With time however, long-lived plasma cells accumulate to normal levels in APRIL-deficient mice ${ }^{16,63}$, indicating a redundant role for BAFF and APRIL or the existence of additional trophic factors for bone marrow plasma cells.

TACI has both positive and negative roles in the regulation of mouse B cells On the one hand, TACI-deficient mice have excessive numbers of B cells, indicating that TACI negatively regulates the size of the B-cell compartment in vivo (reviewed in ${ }^{48}$ ). On the other hand, TACI is required for efficient T-cell-independent type II humoral immune responses ${ }^{48}$. As TACI is strongly expressed by innate B cells (marginal-zone and B1 cells) that are known to participate in T-cell-independent type II immune responses, it is probable that it provides positive signals for the maturation or survival of plasmablasts derived from these innate $\mathrm{B}$ cells ${ }^{64}$.

$\mathrm{Bcma}^{-/-}$mice are healthy, although the survival of long-lived bone-marrow plasma cells is impaired in these mice ${ }^{49}$. BCMA can also promote the APC function of B cells ${ }^{65}$, although BAFF-mediated up-regulation of MHC class II expression through BCMA was also achieved by signalling through TACI and BAFFR ${ }^{20}$.

In summary, studies of deficient mouse strains have shown us that: BAFF and BAFFR control the development and survival of B2 and marginal zone B cells; APRIL regulates aspects of $\mathrm{CD} 40$-independent class switching and promotes plasma 
cell survival; TACI helps humoral responses of innate B cells to repetitive antigens and somehow controls the expansion of the B cell pool; and BCMA contributes to the maintenance of plasma cells. B cell memory, however, does not rely on the BAFF/APRIL system ${ }^{16}$. To what extent these findings in rodents can be extrapolated to humans remains to be determined.

\section{BAFF-mediated survival and growth signals in B cells}

Stimulation of BAFFR potently activates the alternative NF- B2 pathway and weakly activates the classical NF- B1 pathway in primary B cells, whereas TACI is a potent stimulator of the classical NF- B1 pathway (FIG 4) (reviewed in ${ }^{48}$ ). Both NF- B pathways are required for B cell survival, and B cell survival can be sustained by signalling through BAFFR alone ${ }^{48}$. TACI signalling is important for specific B cell types ${ }^{64}$ and could also reinforce BAFFR signals, but the respective contributions of BAFFR and TACI to BAFF-mediated signalling have not been systematically addressed in the studies cited below.

NF- B activation downstream of BAFF has been linked to increased expression of anti-apoptotic proteins, to integrin-mediated localization of B cells in the marginal zone, and to T-cell-independent antibody class switching (reviewed in ${ }^{48}$ ). BAFF not only induces the survival of B cells, but also promotes glycolysis, protein synthesis and cell growth, in great part through the activation of the kinase mammalian target of rapamycin (mTOR) in the context of mTOR complex 1 (mTORC1) [G] ${ }^{66,67}$ (FIG 4). The kinase AKT (also known as PKB) is an important intermediate between BAFF and mTOR. Briefly, BAFF activates phosphoinositide 3kinase (PI3K) through a pathway that remains to be defined. This results in the accumulation of the lipid phosphatidylinositol-3, 4, 5-trisphosphate (PIP3) and membrane recruitment of phosphoinositide-dependent protein kinase 1 (PDK1) and of AKT. AKT is phosphorylated at Thr308 by PDK1, and at a second activation site (Ser473) by protein kinase C \# (PKC\#) ${ }^{66}$ and/or by mTORC2 ${ }^{67}$. Active AKT relieves mTORC1 inhibition. Two well-characterized targets of mTORC1 are p70-S6 Kinase 1 (S6K1) and the eukaryotic initiation factor 4E (eIF4E) binding protein 1 (4EBP1). Phosphorylated S6K1 activates ribosomes and increases the capacity for protein synthesis, whereas phosphorylated 4EBP1 releases eiF4E, which promotes mRNA translation ${ }^{66} .4 \mathrm{EBP} 1$ is also a target of PIM2, a kinase that is induced by NF-
B2 ${ }^{68}$. Simultaneous inactivation of mTORC1 (with rapamycin) and PIM2 (in Pim2 mice, which otherwise have normal B cells) rendered B cells unresponsive to BAFF in terms of both survival and growth, implying that these two kinases are involved in BAFF-mediated signals ${ }^{67}$, although independent clarification of the role of PIM2 is required. The effect on growth in Pim $^{-/}$treated with rapamycin could therefore be explained by inefficient protein synthesis in the combined absence of PIM2 and mTORC1, whereas lack of survival correlates with downregulation of myeloid cell leukaemia sequence 1 (MCL1), as detailed below.

MCL1 is a short-lived member of the BCL-2 family that is required for the development of haematopoietic stem cells and for the maintenance of peripheral B and $\mathrm{T}$ cells ${ }^{69,}{ }^{70}$. The PI3K-AKT pathway (downstream of BAFF) positively regulates transcription of the $\mathrm{Mcll}$ gene ${ }^{71}$. In addition, active eiF4E promotes efficient translation of Mcll mRNA (as well as other mRNAs containing a GC-rich

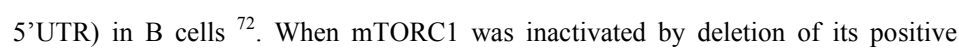
regulator tuberous sclerosis protein 2 (TSC2), B-cell tumours failed to grow as a result of lack of MCL1 expression ${ }^{72}$. In addition, MCL1-deficient B cells do not survive in response to $\mathrm{BAFF}{ }^{67}$, demonstrating the crucial involvement of MCL1 in BAFF-mediated survival. The pro-survival role of other BCL-2-family members, such as the NF- B targets Bcl- $\mathrm{X}_{\mathrm{L}}$ or $\mathrm{A} 1$ in the response to $\mathrm{BAFF}$, is less clearly established. MCL1 acts by inhibiting the pro-apoptotic protein BIM, an essential mediator of B-cell death ${ }^{73}$. Finally, and in agreement with the negative regulation that BAFF and AKT impose on glycogen synthase kinase 3\# (GSK3\#) and protein kinase C (PKC), these two kinases destabilize MCL1 ${ }^{74,75}$. It is also noteworthy that both AKT and PIM2 inactivate the pro-apoptotic factor BAD by phosphorylation.

MYC, by regulating the expression of numerous genes involved in metabolism, is also involved in the control of B-cell growth and renders B cells resistant to atrophy $[\mathbf{G}]^{76}$. Interestingly, MYC is a direct target of the classical NF- B pathway and its expression also requires PI3K activity ${ }^{76}$, providing an additional link between BAFF signalling and cell growth.

In conclusion, the activation of the alternative NF- B and PI3K-AKT-mTOR pathways, and the upregulation of expression of Mcl1 provide a rational basis for the effects of BAFF on B-cell survival and metabolic fitness. 


\section{BAFF, APRIL and B-cell homeostasis and tolerance}

As a key survival factor for late transitional B cells ${ }^{53}$, BAFF has a crucial role in Bcell homeostasis. APRIL has no or little role in this respect: its excessive production leads to the development of B1 B-cell lymphomas in aging April-transgenic mice, but it does not cause autoimmunity and has no immediate effect on B-cell homeostasis (reviewed in ${ }^{4}$ ). For reasons that are still not fully clarified, B-cell homeostasis depends on BAFF produced by radio-resistant cells rather than bone-marrow-derived cells ${ }^{55}$.

BAFFR, by transmitting survival signals, is a positive regulator of B-cell homeostasis, whereas signalling through TACI decreases the size of the B-cell pool. To do so, TACI could directly transmit inhibitory or apoptotic signals to B cells ${ }^{48,77}$, or it could indirectly regulate the B-cell pool by controlling the amount of BAFF that is available for signalling through BAFFR ${ }^{20}$ or potentially the amount of TRAF molecules available to transmit BAFFR signals. There is also evidence that $\mathrm{B}$ cells exposed to TLR agonists upregulate BAFFR and TACI ${ }^{78}$, which upon BAFF signalling somehow induce CD95 (FAS) expression, sensitizing B cells to CD95mediated killing ${ }^{79}$. The relative contributions of these different pathways to the negative regulatory effect of TACI on the B-cell pool remain to be determined.

In a physiological situation, strongly self-reactive B cells compete with 'healthy' B cells for survival factors and for entry in the niche provided by the B-cell follicle in lymphoid tissue (reviewed in ${ }^{80}$ ). As BAFF promotes B-cell survival, it overexpression could potentially break B-cell tolerance by rescuing self-reactive B cells from deletion. However, the use of animal models mimicking the natural competition between healthy and self-reactive $\mathrm{B}$ cells has not revealed any catastrophic breakdown of B-cell tolerance or massive escape of high-affinity autoreactive B cells in Baff-transgenic mice ${ }^{81,82}$. In fact, strongly self-reactive B cells are deleted, anergized or undergo receptor editing ${ }^{1}$ before they start expressing BAFFR on the cell surface ${ }^{82}$, explaining the weak effect of BAFF on these cells. However, healthy B cells and low-affinity self-reactive B cells require a basal BCR signal to be positively selected ${ }^{83-85}$. The nature of this basal BCR signal remains unclear but was proposed because deletion of the BCR in mature B cells led to their loss ${ }^{84}$. [AU: the relevance of a basal BCR signal to BAFF signalling is not clear here]. So, the effect of excess BAFF seems to be on the positive selection and increased proliferation of low-affinity self-reactive B cells, mostly marginal-zone B cells ${ }^{53,82}$. In conclusion, the nature of the $\mathrm{BCR}$ on developing self-reactive $\mathrm{B}$ cells, the quality of its interaction with self-antigen and the need to compete with healthy $B$ cells are key determinants of whether excess BAFF will allow emergence of these cells into the mature B cell pool ${ }^{81,82,86,87}$. Current data suggest that excess BAFF mainly supports the survival of low-affinity self-reactive B cells.

\section{The role of BAFF in autoimmunity}

Baff-transgenic mice develop severe autoimmune symptoms that are similar to systemic lupus erythematosus (SLE) [G] and Sjögren's syndrome in human (reviewed in ${ }^{31}$ ). However, as excessive BAFF production seems to support the survival of low-affinity self-reactive B cells in a complex B-cell repertoire, rather than resulting in a breakdown of B-cell tolerance, can that event alone explain the devastating autoimmune disease that develops in Baff-transgenic mice? The current literature on SLE agrees that the disease is mediated by several types of dysregulated immune cell: B cells, through autoantibody production or antigen presentation to $\mathrm{T}$ cells; innate cells such as plasmacytoid DCs, which can produce high levels of stimulatory cytokines such as type I IFN; and T cells, which are key players in the pathogenesis of SLE (reviewed in ${ }^{88,89}$ ). Baff-transgenic mice have more effector T cells, but they also have a larger regulatory $\mathrm{T}$ cell compartment ${ }^{50}$. In any case, $\mathrm{T}$ cells are not responsible for the autoimmune disorder of Baff-transgenic mice, because autoimmunity also develops to the same extent in T-cell-deficient Bafftransgenic mice (FIG 5), the first described animal model of T-cell-independent lupu ${ }^{50}$. T-cell-independent activation of self-reactive B cells is not unique to the Bafftransgenic model, as similar conclusions were reached in a separate mouse model of autoimmunity ${ }^{90}$. Collectively, these findings show that chronic activation or survival of T-cell-independent low-affinity self-reactive B cells by BAFF can lead to a form of SLE. This suggests that some patients with SLE might benefit from B-celldepleting or BAFF-antagonist therapies.

TLR signalling promotes BAFF effects through TACI.

In Baff-transgenic mice, B1 B-cell activity is associated with nephritis, whereas marginal-zone B cells might have a role in the destruction of the salivary glands but do not infiltrate the inflamed kidneys ${ }^{91,92}$. Baff-transgenic mice lacking marginalzone B cells no longer developed Sjögren's-like syndromes but they still developed 
severe nephritis ${ }^{92}$. However, nephritis did not develop in Baff-transgenic mice reconstituted with MYD88-deficient B cells ${ }^{50}$, which indicates that activation of MYD88, possibly downstream of TLRs, leads to the production of pro-inflammatory autoantibodies that elicit complement activation and inflammation in the kidney ${ }^{50}$ (FIG 5). TACI might provide the link between BAFF overexpression and MYD88dependent activation of B cells. Indeed, activation of TLR7 and TLR9 strongly upregulated TACI expression ${ }^{50,78,93,94}$, a receptor that is thought to provide positive signals for the maturation or the survival of plasmablasts in T-cell-independent type II antibody responses (reviewed in ${ }^{48}$ ). These results suggest a tight connection between TLR activation and TACI expression, which might be at the centre of the pathogenic mechanism in Baff-transgenic mice (FIG 5).

In summary, the link between TLRs and TACI on B cells could be important for T-cell-independent antibody responses and, if dysregulated, result in SLE symptoms.

\section{The role of BAFF and APRIL in human diseases}

The Baff-transgenic mouse model showed a correlation between excess BAFF and autoimmunity (reviewed in ${ }^{31}$ ). Increased levels of BAFF have been measured in patients suffering from various autoimmune conditions and a correlation with disease progression has frequently been observed (reviewed in ${ }^{60}$ ). However, it is unclear whether increased levels of BAFF are a primary cause of autoimmunity or if autoimmunity is the result of increased production of inflammatory cytokines such as IFN, which are known to promote BAFF production. The role of APRIL in human autoimmunity is unclear and its circulating levels do not parallel those of BAFF.

Increased levels of BAFF and APRIL in lymphoid cancers are a wellestablished observation (reviewed in ${ }^{95}$ ). Lymphoid cancerous cells can produce BAFF as an autocrine survival factor, and APRIL can have a similar role. Interestingly, BAFF expression has recently been detected in non-lymphoid breast cancer cells from epithelial origin ${ }^{43}$, which indicates that the role of BAFF in cancer might be wider than first thought. BAFF levels are also increased in inflammation, allergy and viral infection, possibly as a consequence of the production of BAFF inducers such as IFN. This might, in part, explain the occasional autoimmune symptoms that are associated with some viral infections. Thus, the correlation observed between dysregulation of BAFF and APRIL production and disease in humans has extended from autoimmunity and lymphoid cancers to other pathologies, and more work will be required to understand the role of BAFF and APRIL in these diseases.

\section{BAFF and APRIL inhibitors in the clinic: benefits and challenges.}

The relevance of BAFF in mouse models of autoimmunity, the implication of BAFF and/or APRIL in the survival of lymphoma cells ex vivo and the measure of increased BAFF levels in various pathologies has prompted several clinical trials with BAFF and APRIL antagonists. The most advanced clinical program is led by Human Genome Sciences (HGS) in partnership with Glaxo Smith Kline (GSK). The product, a fully human BAFF-specific monoclonal antibody (Lymphostat-B ${ }^{\circledR}$ (Belimumab) ${ }^{96}$, has been tested in completed phase II clinical trials in patients with rheumatoid arthritis and SLE ${ }^{97}$. HGS and GSK have also agreed to test Lymphostat-B in patients with multiple sclerosis as increased levels of BAFF have been detected in multiplesclerosis lesions ${ }^{44}$ and recent results from a phase II clinical trial with rituximab (a CD20-specific B-cell-depleting antibody) indicated clinical benefits from B-cellspecific therapies in multiple sclerosis ${ }^{98}$.

The second program is led by ZymoGenetics Inc. in partnership with MerckSerono and is testing a TACI-Fc fusion protein (Atacicept), which unlike Lymphostat-B blocks both BAFF and APRIL ${ }^{99}$. Phase II/III clinical trials are currently in progress for SLE, lupus nephritis, rheumatoid arthritis and relapsing multiple sclerosis. Other haematological conditions are being tested in phase clinical trials. In general, Atacicept is well tolerated ${ }^{100}$ and its safety profile is good. Atacicept seems to be particularly promising for the treatment of SLE and rheumatoid arthritis, and it could help patients who are not responding to TNF antagonists.

An important question is whether the targeting of B cells through the BAFF/APRIL system will be as efficient as currently approved treatments for rheumatoid arthritis. Indeed, TNF antagonists and CD20-specific agents have set the bar very high, and the lacklustre results from the Lymphostat-B phase II clinical trial in rheumatoid arthritis indicate that inhibition of BAFF alone might not compare favourably with currently used treatments for rheumatoid arthritis. A caveat (or advantage) of blocking BAFF is to target mainly precursor B cell subpopulations rather than established antibody-producing clones or B cell memory ${ }^{56}$ [AU: please 
explain the implications of this]. Eliminating de novo production of self-reactive B cells is critical, yet in some disease settings elimination of established long-lived memory self-reactive B cells or autoantibody-producing cells may be as important. Another issue is the difference between human and mouse B cells in terms of the requirement of BAFF for survival. BAFF inhibition in non-human primates does not affect the survival of putative transitional B cells, is less potent at eliminating peripheral mature B cells, and has little effect on the survival and function of terminally differentiated plasma cells when compared with mice ${ }^{101,102}$. This could depend on BAFF-independent B cell survival niches such as that described in the extravascular compartment of the bone marrow surrounding vascular sinusoids [G] (reviewed in ${ }^{103}$ ). Nevertheless, it seems that use of the BAFF-specific inhibitor Lymphostat-B in patients with SLE might be a promising therapeutic avenue.

Blocking BAFF, APRIL and their heteromers with Atacicept rather than with BAFF-specific inhibitors could have a different outcome in terms of plasma cells. Indeed, APRIL is implicated in plasma-cell survival and antibody production in mice 16, 49 and humans ${ }^{5}$. In addition, mutations in both TACI alleles always result in antibody deficiency (Box 1), which indicates that blocking both TACI ligands might be more effective at eliminating antibody-producing cells in humans than are BAFF inhibitors or CD20-specific therapies, which are poorly efficacious on plasma cells.

The treatment of patients with multiple sclerosis with BAFF and/or APRIL inhibitors is an attractive avenue considering the potential role of B cells in some patients. Moreover, currently approved IFN\# treatments for multiple sclerosis and Bcell-depleting agents in patients with autoimmune conditions are both known to increase serum BAFF levels ${ }^{104,105}$, raising concerns about potential B-cell-specific autoimmune problems associated with these treatments, and suggesting in both cases that combining existing treatments with BAFF inhibitors could improve the outcome.

\section{Conclusions and perspective}

The past decade of studying BAFF and APRIL has led to one of the most important paradigm shifts in B-cell immunology. BAFF has emerged as a cytokine that is as important as the BCR in deciding the life and death of a maturing B cell, and important progress has been made in understanding BAFF signalling in B cells. Recent work has also revealed an unappreciated specific role of B cells and innate immune mechanisms in autoimmunity, controlled by BAFF. The characterization of different biochemical forms of BAFF and APRIL has added a new layer of signalling possibilities through one given receptor, in particular through TACI, which are likely to explain differences in receptor signalling and subsequent biology. Finally, the recent realisation that BAFF, APRIL and their receptors are expressed on a wide array of immune and non-immune cell types is opening a new door of exciting biological possibilities. Development of BAFF and/or APRIL inhibitors in the clinic is still strong and ongoing. The main challenge will be to understand better the differences in the BAFF/APRIL system in humans compared with mice and to obtain therapeutic benefits superior to those of currently approved treatments. The future might also show the advantage of combining existing therapies with BAFF and/or APRIL inhibition.

\section{Acknowledgements}

We thank S. Gardam for the design of FIG. 2. This work was funded by grants from The National Health and Medical Research Council (NHMRC) of Australia, the New South Wales Lupus association and the Nancy E Pendergast Charitable Trust Fund Perpetual (to F.M.), and the Swiss National Science Foundation (to P.S.). We apologise to the many authors who have contributed to this field but have not been cited in this manuscript due to referencing restriction.

\section{References}

1. Goodnow, C.C., Sprent, J., Fazekas de St Groth, B. \& Vinuesa, C.G. Cellular and genetic mechanisms of self tolerance and autoimmunity. Nature 435, 590597 (2005).

2. Hardy, R.R. \& Hayakawa, K. B cell development pathways. Ann. Rev Immunol. 19, 595-621 (2001).

3. Anderson, S.M., Tomayko, M.M., Ahuja, A., Haberman, A.M. \& Shlomchik, M.J. New markers for murine memory B cells that define mutated and unmutated subsets. J. Exp. Med. 204, 2103-2114 (2007)

4. Dillon, S.R., Gross, J.A., Ansell, S.M. \& Novak, A.J. An APRIL to remember: novel TNF ligands as therapeuthic targets. Nature Rev. Drug Disc. 5, 235-246 (2006).

5. Huard, B. et al. APRIL secreted by neutrophils binds to heparan sulfate proteoglycans to create plasma cell niches in human mucosa. J. Clin. Invest. 118, 2887-2895 (2008)

This study highlights the relevance of APRIL binding to proteoglycans in vivo and in humans. 
6. Schwaller, J. et al. Paracrine promotion of tumor development by the TNF ligand APRIL in Hodgkin's Disease. Leukemia 21, 1324-1327 (2007).

7. Li, X. et al. Immune opsonins modulate BLyS/BAFF release in a receptorspecific fashion. J. Immunol. 181, 1012-1018 (2008).

8. Liu, Y. et al. Ligand-receptor binding revealed by the TNF family member TALL-1. Nature 423, 49-56 (2003).

This study showed that twenty trimers of BAFF assemble in a capsid-like structure in which the receptor-binding sites remain exposed and ac

9. Bossen, C. \& Schneider, P. BAFF, APRIL and their receptors: structure, function and signaling. Semin. Immunol. 18, 263-275 (2006).

10. Hymowitz, S.G. et al. Structures of APRIL-receptor complexes: like BCMA TACI employs only a single cysteine-rich domain for high affinity ligand binding. J. Biol. Chem. 280, 7218-7227 (2005)

11. Hendriks, J. et al. Heparan sulfate proteoglycan binding promotes APRILinduced tumor cell proliferation. Cell Death Differ. 12, 637-648 (2005).

12. Ingold, K. et al. Identification of proteoglycans as the APRIL-specific binding partners. J. Exp. Med. 201, 1375-1383 (2005).

13. Kimberley, F.C. et al. The proteoglycan (heparan sulfate proteoglycan) binding domain of APRIL serves as a platform for ligand multimerization and cross-linking. FASEB J. 23, 1-12 (2009).

14. Bischof, D. et al. Selective activation of TACI by syndecan-2. Blood 107, 3235-3242 (2006).

15. Sakurai, D. et al. TACI regulates IgA production by APRIL in collaboration with HSPG. Blood 109, 2961-2967 (2007).

16. Benson, M.J. et al. Cutting Edge: The Dependence of Plasma Cells and Independence of Memory B Cells on BAFF and APRIL. J. Immunol. 180, 3655-3659 (2008)

17. Day, E.S. et al. Selectivity of BAFF/BLyS and APRIL for binding to the TNF family receptors BAFFR/BR3 and BCMA. Biochemistry 44, 1919-193 (2005)

18. Castigli, E. et al. TACI is mutant in common variable immunodeficiency and IgA deficiency. Nature Genet. 37, 829-834 (2005).

19. Salzer, U. et al. Mutations in TNFRSF13B encoding TACI are associated with common variable immunodeficiency in humans. Nature Genet. 37, 820-828 (2005).

20. Bossen, C. et al. TACI, unlike BAFF-R, is solely activated by oligomeric BAFF and APRIL to support survival of activated B cells and plasmablasts. Blood 111, 1004-1012 (2008).

An in vitro demonstration that trimeric forms of $\mathrm{BAFF}$ and APRIL bind to in vitroder to the receptor TACI, but that only miths Cha

W. \& Jelinek, D.F. A role for BLyS in the activation of innate immune cells. Blood 108, 2687-2694 (2006).

22. Chang, S.K., Mihalcik, S.A. \& Jelinek, D.F. B lymphocyte stimulator regulates adaptive immune responses by directly promoting dendritic cell maturation

23. Ye, H. \& Wu, H. Thermodynamic characterization of the interaction between TRAF2 and TNFR peptides by isothermal titration calorimetry. Proc. Natl. Acad. Sci. U S A 97, 8961-8966 (2000)
24. Baud, V. et al. Signaling by proinflammatory cytokines: oligomerization of TRAF2 and TRAF6 is sufficient for JNK and IKK activation and target gene induction via an amino-terminal effector domain. Genes Dev. 13, 1297-1308 (1999).

25. Gardam, S., Sierro, F., Basten, A., Mackay, F. \& Brink, R. TRAF2 and TRAF3 signal adapters act cooperatively to control the maturation and survival signals delivered to B cells by the BAFF receptor. Immunity 28, 391 401 (2008)

26. Xie, P., Stunz, L.L., Larison, K.D., Yang, B. \& Bishop, G.A. Tumor necros factor receptor-associated factor 3 is a critical regulator of B cell homeostasi in secondary lymphoid organs. Immunity 27, 253-267 (2007).

27. Xu, L.-G. \& Shu, H.-B. TNFR-associated factor-3 is associated with BAFF-R and negatively regulates BAFF-R-mediated NF- B activation and IL-10 production. J. Immunol. 169, 6883-6889 (2002).

28. Liao, G., Zhang, M., Harhaj, E.W. \& Sun, S.C. Regulation of the NF-kappaBinducing kinase by tumor necrosis factor receptor-associated factor 3-induced degradation. J. Biol. Chem. 279, 26243-26250 (2004).

29. Matsuzawa, A. et al. Essential cytoplasmic translocation of a cytokine receptor-assembled signaling complex. Science 321, 663-668 (2008).

30. Vallabhapurapu, S. et al. Nonredundant and complementary functions of TRAF2 and TRAF3 in a ubiquitination cascade that activates NIK-dependent alternative NF-kappaB signaling. Nature Immunol. 9, 1364-1370 (2008).

31. Mackay, F., Schneider, P., Rennert, P. \& Browning, J.L. BAFF and APRIL: a tutorial on B cell survival. Ann. Rev. Immunol. 21, 231-264 (2003).

32. Scapini, P., Bazzoni, F. \& Cassatella, M.A. Regulation of B-cell-activating factor (BAFF)/B lymphocyte stimulator (BLyS) expression in huma neutrophils. Immunol. Lett. 116, 1-6 (2008).

33. Boule, M.W. et al. Toll-like Receptor 9-Dependent and -Independen Dendritic Cell Activation by Chromatin-Immunoglobulin G Complexes. Exp. Med. 199, 1631-1640 (2004).

34. Mackay, F. \& Leung, H. The role of the BAFF/APRIL system on T cell function. Semin. Immunol. 18, 284-289 (2006).

35. Chu, V.T., Enghard, P., Riemekasten, G. \& Berek, C. In vitro and in vivo activation induces BAFF and APRIL expression in B cells. J. Immunol. 179, 5947-5957 (2007).

36. Kern, C. et al. Involvement of BAFF and APRIL in the resistance to apoptosis of B-CLL through an autocrine pathway. Blood 103, 679-688 (2004).

37. Langat, D.L., Wheaton, D.A., Platt, J.S., Sifers, T. \& Hunt, J.S. Signaling pathways for B cell-activating factor (BAFF) and a proliferation-inducing ligand (APRIL) in human placenta. Am. J. Pathol. 172, 1303-1311 (2008).

38. Alsaleh, G. et al. BAFF synthesis by rheumatoid synoviocytes is positively controlled by alphasbetal integrin stimulation and is negatively regulated by tumor necrosis factor alpha and toll-like receptor ligands. Arthritis Rheum. 56, tumor necrosis fact

39. Abe, M. et al. BAFF and APRIL as osteoclast-derived survival factors for myeloma cells: a rationale for TACI-Fc treatment in patients with multiple myeloma. Leukemia 20, 1313-1315 (2006)

40. Geffroy-Luseau, A., Jego, G., Bataille, R., Campion, L. \& Pellat-Deceunynck, C. Osteoclasts support the survival of human plasma cells in vitro. Int Immunol. 20, 775-782 (2008). 
41. Kato, A., Truong-Tran, A.Q., Scott, A.L., Matsumoto, K. \& Schleimer, R.P. Airway Epithelial Cells Produce B Cell-Activating Factor of TNF Family by an IFN-beta-Dependent Mechanism. J. Immunol. 177, 7164-7172 (2006).

42. Ittah, M. et al. Viruses induce high expression of BAFF by salivary gland epithelial cells through TLR- and type-I IFN-dependent and -independent epithelial cells through TLR- and type-I
pathways. Eur. J. Immunol. 39, 1-9 (2008).

43. Pelekanou, V. et al. Expression of TNF-superfamily members BAFF an APRIL in breast cancer: Immunohistochemical study in 52 invasive ductal breast carcinomas. BMC Cancer 8, $76(2008)$.

44. Krumbholz, M. et al. BAFF is produced by astrocytes and up-regulated in multiple sclerosis lesions and primary central nervous system lymphoma. $J$. Exp. Med. 201, 195-200 (2005).

45. Thangarajh, M., Masterman, T., Hillert, J., Moerk, S. \& Jonsson, R. A proliferation-inducing ligand (APRIL) is expressed by astrocytes and is increased in multiple sclerosis. Scand. J. Immunol. 65, $92-98$ (2007).

46. Darce, J.R., Arendt, B.K., Wu, X. \& Jelinek, D.F. Regulated Expression of BAFF-Binding Receptors during Human B Cell Differentiation. J. Immunol. 179, 7276-7286 (2007).

47. Ng, L.G. et al. BAFF-R is the principal BAFF receptor facilitating BAFF costimulation of B and T cells. J. Immunol. 173, 807-817 (2004).

48. Mackay, F. \& Schneider, P. TACI, an enigmatic BAFF/APRIL receptor, with new unappreciated biochemical and biological properties. Cytokine Growth Factor Rev. 19, 263-276 (2008).

49. O'Connor, B.P. et al. BCMA is essential for the survival of long-lived bone marrow plasma cells. J. Exp. Med. 199, 91-97 (2004).

50. Groom, J.R et al. BAFF and MyD88 signals promote a lupuslike disease Groom, J.R. et al. BAFF and MyD88 signals promote a
independent of T cells. J. Exp. Med. 204, 1959-1971 (2007).

Allonity in Baff-transgenic mice is T cell-independent. Several lines of evidence indicate that autoimmunity correlates with reciprocal

\section{induction and activation of TACI and TLRs on innate B cells.}

51. Allman, D., Srivastava, B. \& Lindsley, R.C. Alternative route to maturity: branch points and pathways for generating follicular and marginal zone $\mathrm{B}$ cells. Immunol. Rev. 197, 147-160 (2004).

52. Pillai, S., Cariappa, A. \& Moran, S.T. Marginal zone B cells. Annu. Rev. Immunol. 23, 16-19 (2005).

53. Meyer-Bahlburg, A., Andrews, S.F., Yu, K.O., Porcelli, S.A. \& Rawlings, D.J. Characterization of a late transitional B cell population highly sensitive to BAFF-mediated homeostatic proliferation. J. Exp. Med. 205, 155-168 (2008).

54. Crowley, J.E et al. Homeostatic control of B lymphocyte subsets. Immunol. Res. 42, 75-83 (2008).

55. Gorelik, L. et al. Normal B cell homeostasis requires B cell activation factor production by radiation-resistant cells. J. Exp. Med. 198, 937-945 (2003).

56. Scholz, J.L. et al. BLyS inhibition eliminates primary B cells but leaves natural and acquired humoral immunity intact. Proc. Natl. Acad. Sci. US A natural and acquired hum

57. Hardenberg, G., van Bostelen, L., Hahne, M. \& Medema, J.P. Thymu independent class switch recombination is affected by APRIL. Immunol. Cell. Biol. 86, 530-534 (2008)
58. Belnoue, E. et al. APRIL is critical for plasmablast survival in the bone marrow and poorly expressed by early-life bone marrow stromal cells. Blood 111, 2755-2764 (2008)

59. Castigli, E. et al. TACI and BAFF-R mediate isotype switching in B cells. $J$. Exp. Med. 201, 35-39 (2005).

60. Mackay, F., Sierro, F., Grey, S.T. \& Gordon, T.P. The BAFF/APRIL system: an important player in systemic rheumatic diseases. Curr. Dir. Autoimmun. 8

61. He, B. et al. Intestinal bacteria trigger $\mathrm{T}$ cell-independent immunoglobulin $\mathrm{A}(2)$ class switching by inducing epithelial-cell secretion of the cytokine APRIL. Immunity 26, 812-826 (2007).

62. Massacand, J.C. et al. Intestinal bacteria condition dendritic cells to promote IgA production. PLOS ONE 3, e2588 (2008).

63. Varfolomeev, E. et al. APRIL-deficient mice have normal immune system development. Mol. Cell. Biol. 24, 997-1006 (2004).

64. Mantchev, G.T., Cortesao, C.S., Rebrovich, M., Cascalho, M. \& Bram, R.J. TACI is required for efficient plasma cell differentiation in response to Tindependent type 2 antigens. J. Immunol. 179, 2282-2288 (2007).

65. Yang, M. et al. B cell maturation antigen, the receptor for a proliferationinducing ligand and B cell-activating factor of the TNF family, induces antigen presentation in B cells. J Immunol 175, 2814-2824 (2005).

66. Patke, A., Mecklenbrauker, I., Erdjument-Bromage, H., Tempst, P. \& Tarakhovsky, A. BAFF controls B cell metabolic fitness through a PKC betaand Akt-dependent mechanism. J. Exp. Med. 203, 2551-2562 (2006).

67. Woodland, R.T. et al. Multiple signaling pathways promote B lymphocyte stimulator dependent B-cell growth and survival. Blood 111, $750-760$ (2008)

References 66 and 67 show the essential roles of the kinases mTOR and PIM2 in mediating the cellular growth and survival effects of BAFF on B cells. 68 . Enzler, T. et al. Alternative and classical NF-kappa B signaling retain autoreactive B cells in the splenic marginal zone and result in lupus-like disease. Immunity 25, 403-415 (2006)

69. Opferman, J.T. et al. Obligate role of anti-apoptotic MCL-1 in the survival of hematopoietic stem cells. Science 307, 1101-1104 (2005).

70. Opferman, J.T. et al. Development and maintenance of B and T lymphocytes requires antiapoptotic MCL-1. Nature 426, 671-676 (2003).

71. Wang, J.M. et al. The antiapoptotic gene mcl-1 is up-regulated by the phosphatidylinositol 3-kinase/Akt signaling pathway through a transcription factor complex containing CREB. Mol. Cell Biol. 19, 6195-6206 (1999).

72. Mills, J.R. et al. mTORC1 promotes survival through translational control of Mcl-1. Proc. Natl. Acad. Sci. U S A 105, 10853-10858 (2008).

73. Bouillet, $\mathrm{P}$ et al. Proapoptotic Bcl-2 relative Bim required for certain apoptotic responses, leukocyte homeostasis, and to prelude autoimmunity. apoptotic responses, leukocyte

74. Maurer, U., Charvet, C., Wagman, A.S., Dejardin, E. \& Green, D.R. Glycogen synthase kinase-3 regulates mitochondrial outer membrane permeabilization and apoptosis by destabilization of MCL-1. Mol. Cell. 21, 749-760 (2006)

75. Sitailo, L.A., Tibudan, S.S. \& Denning, M.F. The protein kinase C delt catalytic fragment targets Mcl-1 for degradation to trigger apoptosis. J. Biol. Chem. 281, 29703-29710 (2006). 
76. Grumont, R.J., Strasser, A. \& Gerondakis, S. B cell growth is controlled by phosphatidylinosotol 3-kinase-dependent induction of Rel/NF-kappaB regulated c-myc transcription. Mol. Cell 10, 1283-1294 (2002).

77. Sakurai, D. et al. TACI attenuates antibody production costimulated by BAFF-R and CD40. Eur. J. Immunol. 37, 110-118 (2007).

78. Katsenelson, N. et al. Synthetic $\mathrm{CpG}$ oligodeoxynucleotides augment BAFFand APRIL-mediated immunoglobulin secretion. Eur. J. Immunol. 37, 17851795 (2007).

79. Acosta-Rodriguez, E.V. et al. BAFF and LPS cooperate to induce B cells to become susceptible to CD95/Fas-mediated cell death. Eur. J. Immunol. 37, 990-1000 (2007)

80. Stadanlick, J.E. \& Cancro, M.P. BAFF and the plasticity of peripheral B cell tolerance. Curr. Opin. Immunol. 20, 158-161 (2008)

81. Ait-Azzouzene, D., Gavin, A.L., Skog, P., Duong, B. \& Nemazee, D. Effect of cell:cell competition and BAFF expression on peripheral B cell tolerance and B-1 cell survival in transgenic mice expressing a low level of Igkappa-reactive macroself antigen. Eur. J. Immunol. 36, 985-996 (2006).

82. Thien, M. et al. Excess BAFF rescues self-reactive B cells from periphera deletion and allows them to enter forbidden follicular and marginal zone niches. Immunity 20, $785-798$ (2004).

This paper shows that BAFF overexpression does not prevent negative This paper shows that BAF overexpression does not prevent negative sects in a complex B-cell

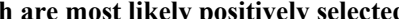

83. Gaudin, E. et al. Positive selection of B cells expressing low densities of selfreactive BCRs. J. Exp. Med. 199, 843-853 (2004).

84. Lam, K.P., kuhn, R. \& Rajewsky, K. In vivo ablation of surface immunoglobulin on mature $\mathrm{B}$ cells by inducible gene targeting results in rapi death. Cell 90, 1073-1083 (1997)

85. Wen, L. et al. Evidence of marginal-zone B cell-positive selection in spleen Immunity 23, 297-308 (2005).

86. Hondowicz, B.D. et al. The role of BLyS/BLyS receptors in anti-chromatin B cell regulation. Int Immunol 19, 465-475 (2007).

87. Lesley, R. et al. Reduced competitiveness of autoantigen-engaged B cells due to increased dependence on BAFF. Immunity 20, 441-453 (2004).

88. Hoffman, R.W. T cells in the pathogenesis of systemic lupus erythematosus. Clin Immunol 113, 4-13 (2004).

89. Marshak-Rothstein, A. Toll-like receptors in systemic autoimmune disease. Nature Rev. Immunol. 6, 823-835 (2006).

90. Herlands, R.A., Christensen, S.R., Sweet, R.A., Hershberg, U. \& Shlomchik, M.J. T Cell-Independent and Toll-like Receptor-Dependent Antigen-Driven Activation of Autoreactive B Cells. Immunity 29, 249-260 (2008).

91. Batten, M. et al. TNF deficiency fails to protect BAFF transgenic mice against autoimmunity and reveals a predisposition to B cell lymphomas. J. Immunol. 172, 812-822 (2004)

92. Fletcher, C.A. et al. Development of nephritis but not sialadenitis in autoimmune-prone BAFF transgenic mice lacking marginal zone B cells. Eur J. Immunol. 36, 2504-2514 (2006).

93. Ng, L.G. et al. BAFF costimulation of Toll-like receptor-activated B-1 cells Eur. J. Immunol. 36, 1837-1846 (2006).
94. Treml, L.S. et al. TLR stimulation modifies BLyS receptor expression in follicular and marginal zone B cells. J. Immunol. 178, 7531-7539 (2007).

95. Mackay, F. \& Tangye, S.G. The role of the BAFF/APRIL system in B cell Mackay, \&. The (2004).

Baker, K.P. et al. Generation and characterization of LymphoStat-B, a human monoclonal antibody that antagonizes the bioactivities of B Lymphocyt Stimulator. Arthritis Rheum. 48, 3253-3265 (2003).

97. Ding, C. \& Jones, G. Belimumab Human Genome Sciences/Cambridg Antibody Technology/GlaxoSmithKline. Curr. Opin. Investig. Drugs 7, 464 472 (2006).

98. Hauser, S.L. et al. B-cell depletion with rituximab in relapsing-remitting multiple sclerosis. N. Engl. J. Med. 358, 676-688 (2008).

99. Carbonatto, M. et al. Nonclinical safety, pharmacokinetics, and pharmacodynamics of atacicept. Toxicol. Sci. 105, 200-210 (2008).

100. Munafo, A., Priestley, A., Nestorov, I., Visich, J. \& Rogge, M. Safety, pharmacokinetics and pharmacodynamics of atacicept in healthy volunteers. Eur. J. Clin. Pharmacol. 63, 647-656 (2007).

101. Tangye, S.G., Bryant, V.L., Cuss, A.K. \& Good, K.L. BAFF, APRIL an human B cell disorders. Semin. Immunol. 18, 305-317 (2006).

102. Vugmeyster, Y. et al. A soluble BAFF antagonist, BR3-Fc, decreases peripheral blood B cells and lymphoid tissue marginal zone and follicular B cells in cynomolgus monkeys. Am. J. Pathol. 168, 476-489 (2006).

103. Pillai, S. \& Cariappa, A. The bone marrow perisinusoidal niche for recirculating $B$ cells and the positive selection of bone marrow-derived $B$ lymphocytes. Immunol. Cell Biol. 87, 16-19 (2009).

104. Krumbholz, M. et al. Interferon-beta increases BAFF levels in multiple sclerosis: implications for B cell autoimmunity. Brain 131, 1455-1463 (2008).

05. Pers, J.O. et al. BAFF-modulated repopulation of B lymphocytes in the bloo and salivary glands of rituximab-treated patients with Sjogren's syndrome. Arthritis Rheum. 56, 1464-1477 (2007)

106. Salzer, U. et al. Relevance of biallelic versus monoallelic TNFRSF13B mutations in distinguishing disease-causing from risk-increasing TNFRSF13B variants in antibody deficiency syndromes. Blood 113, 1967-1976 (2008).

107. Garibyan, L. et al. Dominant-negative effect of the heterozygous C104R TACI mutation in common variable immunodeficiency (CVID), J. Clin. Invest. 117, 1550-1557 (2007).

108. Rizzi, M. et al. BAFF receptor expression in CVID patients. Clinical Immunol. 123, S15 (2007)

109. Carey, J.B., Moffatt-Blue, C.S., Watson, L.C., Gavin, A.L. \& Feeney, A.J. Repertoire-based selection into the marginal zone compartment during B cell development. J. Exp. Med 205, 2043-2052 (2008).

110. Wardemann, H., Boehm, T., Dear, N. \& Carsetti, R. B-1a B cells that link the innate and adaptive immune responses are lacking in the absence of the spleen. J. Exp. Med. 195, 771-780 (2002).

111. Teague, B.N et al. Cutting edge: Transitional T3 B cells do not give rise to mature B cells, have undergone selection, and are reduced in murine lupus. $J$ Immunol. 178, 7511-7515 (2007). 
112. Gorelik, L. et al. BAFF regulates $\mathrm{CD} 21 / 35$ and $\mathrm{CD} 23$ expression independently of its B cell survival function. J. Immunol. 172, 762-766 (2004).

113. Shimomura, Y. et al. A unique B2 B cell subset in the intestine. J. Exp. Med. 205, 1343-1355 (2008)

114. Shulga-Morskaya, S. et al. B cell-activating factor belonging to the TNF family acts through separate receptors to support B cell survival and T cellindependent antibody formation. J. Immunol. 173, 2331-2341 (2004).

\section{Box 1: TACI and BAFFR in CVID}

Common variable immunodeficiency (CVID) is characterized by a marked hypogammaglobulinaemia and impaired responses to vaccination, resulting in susceptibility to bacterial infections. CVID is diagnosed after exclusion of other causes of hypogammaglobulinemia and, in contrast to other primary immunodeficiencies, generally develops relatively late in life. About $20 \%$ of patients with CVID have signs of autoimmunity and/or lymphoproliferation ${ }^{106}$. Genetic defects in the T-cell costimulatory molecule inducible T-cell costimulator (ICOS) or in the B-cell co-receptor CD19 are occasionally found in patients with CVID, but not as frequently as TACI mutations, which affect $8 \%$ of patients with CVID. Mutation of both TACI alleles is always associated with insufficient antibody production and, most of the time, with CVID ${ }^{106}$. Heterozygous TACI mutations, in particular $\mathrm{C} 104 \mathrm{R}$ and $\mathrm{A} 181 \mathrm{E}{ }^{107}$, predispose to $\mathrm{CVID}$ rather than causing the disease as shown by the fact that: $2 \%$ of the control population carry a mutant TACI allele ${ }^{106}$; CVID develops most of the time in the absence of TACI mutations; an heterozygous TACI mutation (A181E) did not segregate with disease in one familial case of CVID ${ }^{106}$; and of two brothers with a homozygous TACI mutation (S144X), one was affected with CVID but the other had hypogammaglobulinaemia with no clinical signs of immunodeficiency, indicating that other genetic or environmental factors are required to induce $\mathrm{CVID}^{19}$. Humans lacking TACI expression have increased numbers of $\mathrm{B}$ cells (as observed in $\mathrm{Taci}^{-1}$ mice), but decreased immunoglobulin levels (unlike $\mathrm{TaCl}^{-1-}$ mice), suggesting that TACI has a crucial role in immunoglobulin production in humans but not in mice. One patient lacking transmembrane BAFFR was reported with a severe B-cell lymphopaenia ${ }^{108}$. If it can be confirmed that the mutation causes the phenotype, it could indicate that human B cells, similarly to mouse B cells, strongly rely on BAFF signals.

\section{Figure legends}

FIG. 1. BAFF, APRIL and their receptors.

A) Schematic representation of various forms of BAFF and APRIL, and their binding to BAFFR, TACI and BCMA. BAFF and APRIL are synthesized as membrane-bound proteins that can be released as soluble cytokines upon proteolytic cleavage (triangles). Soluble BAFF exists as homotrimers (3-mer) or as a capsid-like assembly of 20 trimers $(60-$ mer). APRIL binds sulphated side chains of heparan sulphate proteoglycan (HSPG) at a site independent from bona fide receptor binding. TWEPRIL, which contains the entire receptor-binding domain of APRIL, is described as a membrane-bound protein only. The arrows indicate interactions that are able to induce signalling, although BAFF 3-mer can also bind to TACI and BCMA. The dashed arrow indicates a weak affinity.

B) Model of signalling induced by BAFF 3-mer versus higher-order oligomers. TRAFs are trimeric intracellular signalling molecules that are recruited to three receptors held in the correct geometry by a trimeric ligand. Recruitment of TRAF3 to trimeric BAFFR leads to its degradation and releases the inhibition imposed by unbound TRAF3 on the alternative NF- B signalling pathway. By contrast, recruitment of TRAF2 or TRAF6 results in a positive signal (classical NF- B) only when at least two trimeric TRAFs are recruited in close proximity in response to higher-order oligomers of ligand, such as BAFF 60-mer or APRIL cross-linked by HSPG

FIG. 2. The role of TRAF molecules in BAFFR signalling

Left panel: In the absence of BAFF binding to BAFFR, NIK complexes with TRAF2 and TRAF3. TRAF2 recruits cellular inhibitor of apoptosis (c-IAP), which target NIK for degradation by ubiquitination. Right panel: After BAFF binding to BAFFR, TRAF3 is recruited to the receptor and TRAF2 promotes TRAF3 degradation. NIK is free to promote NF- B2 activation, which promotes cell survival.

FIG. 3. BAFF-dependent maturation of mouse B cells. 
B cells develop in the bone marrow. Immature transitional type 1 (T1) B cells expressing a receptor lacking $\mathrm{N}$ regions $(\mathrm{N})$ (the site of random nucleotide addition at the V-D junction) are generated in the fetal liver or in the bone marrow and preferentially develop into marginal zone B cells in the spleen or B1 B cells in the peritoneal cavity ${ }^{109}$. Some B1 B cells also home to the spleen ${ }^{110}$. In the spleen, $\mathrm{N}^{+}$ T1 B cells preferentially develop into mature follicular (Fo) B cells ${ }^{109}$ through the $\mathrm{CD} 21^{\text {int }}$ immature $\mathrm{T} 2 \mathrm{~B}$-cell stage, which is a precursor for both follicular and marginal-zone B cells ${ }^{53}$. T2 cells have also been described in the bone marrow. In the spleen, $\mathrm{T} 3$ immature $\mathrm{B}$ cells are thought to be anergic ${ }^{111}$, and a small population of mature $\mathrm{CD} 21^{\text {low }} \mathrm{CD} 23^{-} \mathrm{IgM}^{+} \mathrm{IgD}^{+} \mathrm{B}$ cells survives in the absence of BAFF ${ }^{112}$. A similar population has been described in the intestine, which in this case requires BAFF for survival ${ }^{113}$. B-cell subsets circled in red require BAFF for development and survival.

\section{FIG. 4: Signal transduction through BAFF-BAFFR}

A model of potential BAFF signalling pathways leading to B-cell survival and metabolic fitness. BAFF mediates B cell growth (gain of volume and protein synthesis, but not cell division) in part by enhancing protein translation. The mTOR complex 1 (mTORC1) is activated by the PI3K-AKT pathway, whereas the kinase PIM2 is induced by the alternative NF-kB2 pathway, although the involvement of PIM2 awaits confirmation in independent studies. mTORC1 and PIM2 seem to have redundant roles in activating translation and cell growth. MYC also promotes cell growth and could be at least partially under the control of BAFF through the classical NF- B1 pathway. In addition to cell growth, BAFF promotes B cell survival by favouring a high ratio of MCL1, an anti-apoptotic member of the BCL-2 family, to BIM, a proapoptotic antagonist of BCL-2 family members. This control is achieved at the transcriptional level (through CREB to enhance Mcll, or through Fox03a to repress Bim) and at the translational level (through eukaryotic elongation factor eiF4E). The NF- B pathway also controls the expression of integrins on marginal zone B cells and regulates T-cell-independent isotype switching (TI switch). See text for details.
FIG. 5: The role of excess BAFF in B-cell tolerance and activation of self-reactive B cells.

Upper panel: strongly self-reactive immature $\mathrm{T} 1 \mathrm{~B}$ cells are killed after binding to self-antigen before expressing sufficient BAFFR on the cell-surface and therefore cannot be rescued from deletion by BAFF. By contrast, self-reactive B cells with low affinity for self-antigen are positively selected, acquire expression of BAFFR on the cell-surface, proliferate in response to increased levels of BAFF and mostly accumulate in the marginal-zone compartment. Lower panel: low-affinity selfreactive $\mathrm{B}$ cells, in particular marginal-zone B cells, are particularly responsive to Toll-like receptor (TLR) activation. B cells expressing rheumatoid factor antibody can bind DNA through cell-surface immunoglobulin. After internalisation of the antibody-DNA complex, the DNA can activate TLR9. DNA-specific and RNAspecific self-reactive B cells could therefore be activated directly through TLR9 and TLR7, respectively. TACI activation up-regulates TLR expression, and TLR activation increases TACI expression. After TLR activation, and in the presence of high levels of BAFF, self-reactive B cells produce pro-inflammatory autoantibodies (in particular, $\operatorname{IgG} 2 \mathrm{~b}$ and $\operatorname{IgG} 2 \mathrm{c}$ ), which deposit in the kidney, and promote complement activation and tissue destruction. 
April-

\section{ransgenic}

Expansion of the regulatory $\mathrm{T}$ cell compartment B1 B-cell neoplasia

Increased survival of $\mathrm{CD} 4^{+}$and $\mathrm{CD} 8^{+} \mathrm{T}$ cells (increase production of IL-2 by $\mathrm{CD} 8^{+} \mathrm{T}$ cells)

Enhanced survival of superantigen-reactive $\mathrm{T}$ cells linked to BCL-2 expression

Increased T-cell proliferation

Decreased percentage of $\mathrm{T}$ cells in peripheral lymph nodes.

Same as Baff/- mice

Decreased lifespan of germinal centres

Table 1: Phenotype of genetically modified mice in the BAFF/APRIL system

\begin{tabular}{|c|c|c|}
\hline $\begin{array}{l}\text { Mouse } \\
\text { model }\end{array}$ & Phenotype & References \\
\hline \multirow{3}{*}{$\begin{array}{l}\text { Ligands } \\
\text { Baff }\end{array}$} & & \multirow{4}{*}{$\begin{array}{l}\text { Reviewed } \\
\text { in }^{31}\end{array}$} \\
\hline & Impaired B-cell maturation beyond the $\mathrm{T} 1$ stage & \\
\hline & $\begin{array}{l}\text { Decreased immunoglobulin levels; decreased T-cell- } \\
\text { dependent and -independent immune responses }\end{array}$ & \\
\hline \multirow{5}{*}{ April $^{\prime-}$} & $\begin{array}{l}\text { Modest increase of allograft survival, improved with a } \\
\text { non-effective low dose of cyclosporin }\end{array}$ & \\
\hline & Impaired class switching to $\operatorname{IgA}$ & \multirow{4}{*}{$\begin{array}{l}\text { Reviewed } \\
\text { in } 4 \\
16,58\end{array}$} \\
\hline & $\begin{array}{l}\text { Impaired tetanus-toxoid-specific plasma-cell survival in } \\
\text { the bone marrow }\end{array}$ & \\
\hline & $\begin{array}{l}\text { Normal survival of nitrophenol (NP)-specific bone- } \\
\text { marrow long-lived plasma cells }\end{array}$ & \\
\hline & $\begin{array}{l}\text { Increased percentage of } \\
\text { effector/memory T cells }\end{array}$ & \\
\hline \multirow[t]{2}{*}{$\begin{array}{l}\text { Baff- } \\
\text { transgenic }\end{array}$} & $\begin{array}{l}\text { B-cell hyperplasia from the transitional type } 2 \text { B-cell } \\
\text { stage }\end{array}$ & \multirow[t]{2}{*}{$\begin{array}{l}\text { Reviewed } \\
\text { in } 34,60 \\
50\end{array}$} \\
\hline & $\begin{array}{l}\text { T-cell-independent but MyD88-dependent } \\
\text { autoimmunity: autoantibodies, glomerulonephritis, } \\
\text { inflammation and destruction of the salivary glands. } \\
\text { Decreased saliva production. B1 B cells in the kidney, }\end{array}$ & \\
\hline
\end{tabular}

Impaired class-switch recombination

$\boldsymbol{A} / \boldsymbol{W} \boldsymbol{y} \boldsymbol{S} \boldsymbol{J} \boldsymbol{S}$ Same as Baff-/ mice although less severe.

BaffR

mutant Autoimmunity developing as mice age (might be Autoimmunity developing as mice age (might be
background dependent as $\mathrm{T}$ cells in these mice are hyperresponsive to activation ex vivo).

Impaired T-cell-dependent antibody responses

Taci $^{-1-} \quad$ B-cell hyperplasia as early as the T1 B-cell stage

Increased rate of B-cell proliferation

Defective T-cell-independent type II antibody responses Autoimmunity, glomerulonephritis, autoantibodies

B-cell lymphomas

Impaired class-switch recombination to IgA

Increased number of $\mathrm{CD}^{+} \mathrm{T}$ cells in Peyer's patches

$\mathrm{Taci}^{-1 /} \mathrm{B}$ cells fail to induce the proliferation of antigenspecific cytotoxic $\mathrm{T}$ cells after transfer to B-celldeficient mice.

$B c m a^{-1-}$

Impaired survival of long-lived bone-marrow plasma 16,49
Reviewed 
cells.

APRIL, a proliferation-inducing ligand; BAFF, B cell-activating factor of the TNF family; BAFFR, BAFF receptor; BCMA, B cell maturation antigen; IL-1, interleukin-1; MyD88, myeloid differentiation primary response gene 88; TACI, transmembrane activator and calcium-modulator and cyclophilin ligand (CAML) interactor.

\section{Glossary}

B-cell receptor editing. Process of somatic mutations that selectively alters the sequence and specificity of the $\mathrm{B}$ cell receptor.

Trans-splicing. Splicing of a pre-mRNA spanning two adjacent genes.

Alternative NF- B2 pathway. Signalling cascade resulting in the NF- B essential modulator (NEMO)-independent activation of the transcription factor NF- B Hallmarks of this pathway are the implication of NF- B-inducing kinase (NIK) and the processing of NF- B2 from a 100 precursor to a p52 fragment.
Classical NF- B1 pathway. NEMO-dependent signalling pathway that activates the transcription factor NF- B, which usually contains the $\mathrm{p} 50$ subunit derived from the p105 NF- B1 precursor.

Cytotrophoblasts. Cells of the inner layer of the trophoblast, the outermost layer in an embryo that serves to anchor the embryo to the maternal endometrium.

Marginal-zone B cells. B cells that reside in the marginal zone, a region at the interface between the non-lymphoid red pulp and the lymphoid white-pulp of the spleen

B1 B cells. Sub-class of B cells residing predominantly in the peritoneal and pleural cavities, that express $\mathrm{IgM}$ in greater quantities than $\mathrm{IgG}$ and whose receptors are polyspecific (have low affinities for many different antigens)

Class switching. The somatic-recombination process by which immunoglobulin isotypes are switched from IgM to IgG, IgA or IgE. This imparts flexibility to the humoral immune response and allows it to exploit the different capacities of these antibody classes to activate the appropriate downstream effector mechanisms.

Heparitinase. An enzyme that catalyses the eliminative cleavage of glycosidic bonds in heparan sulphate.

T-cell-independent type II response. Type of B cell activation in which several of the same antigen are presented in a way that causes cross-linking of antibodies on the surface of B cells, without requiring $\mathrm{T}$ cell help.

mTORC1 complex. Multiprotein complex comprising mTOR that functions as a nutrient/energy/redox sensor and controls protein synthesis. The activity of this complex is stimulated, among others, by insulin, growth factors and amino acids.

Atrophy. Cellular wasting resulting in volume loss and decreased protein content. It is the opposite of cell growth. 
Systemic lupus erythematosus (SLE). An autoimmune disease in which autoantibodies that are specific for DNA, RNA or proteins associated with nucleic acids form immune complexes that damage small blood vessels, especially in the kidney. Patients with SLE generally have abnormal B- and T-cell function.

Vascular sinusoids. Specialized blood vessels in haematopoietic tissues through which venous circulation occurs and that have thin walls formed by a discontinuous, irregularly-shaped endothelium that allows cells to pass in and out of circulation. 


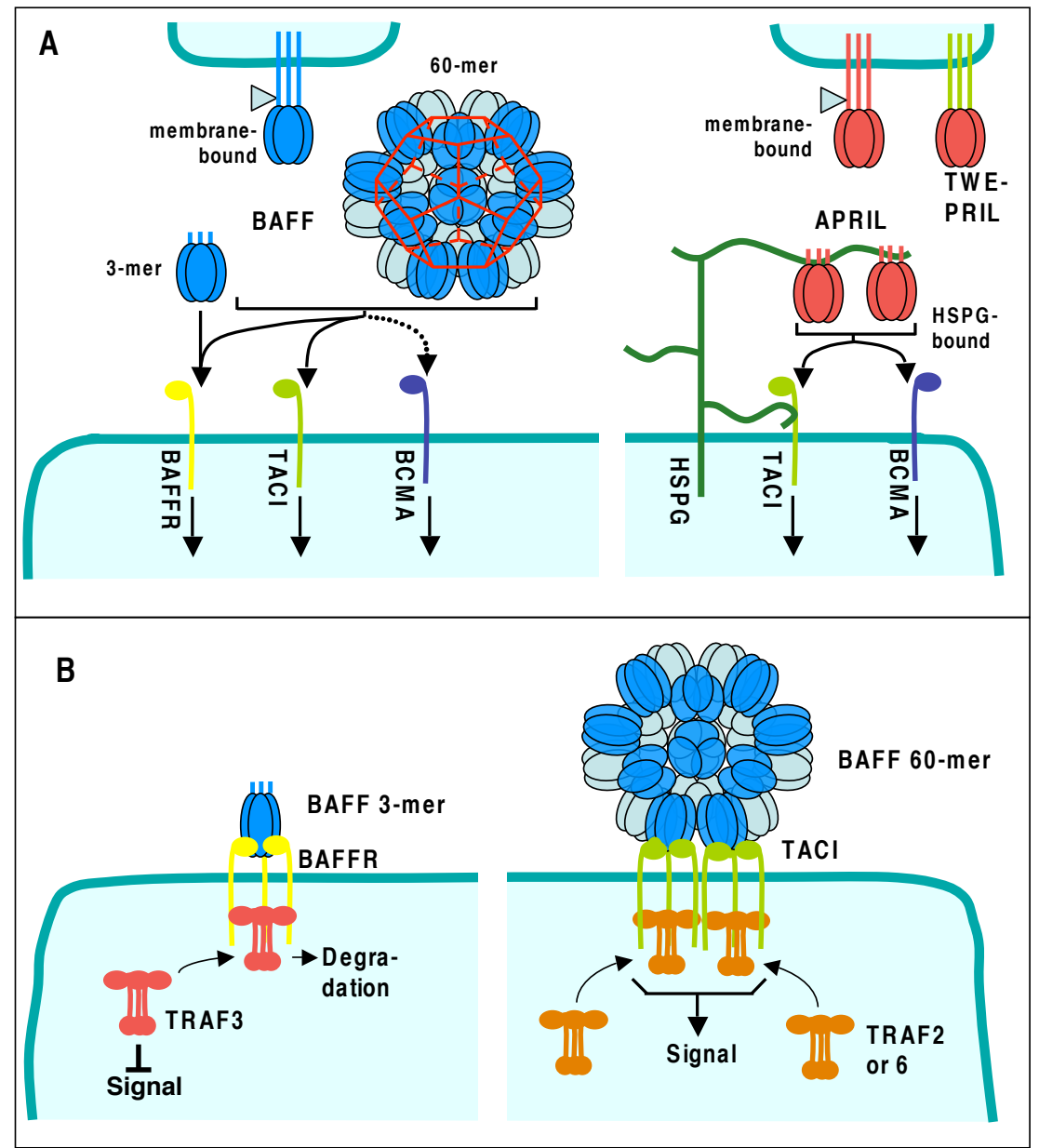

Mackay et al, Fig 1 

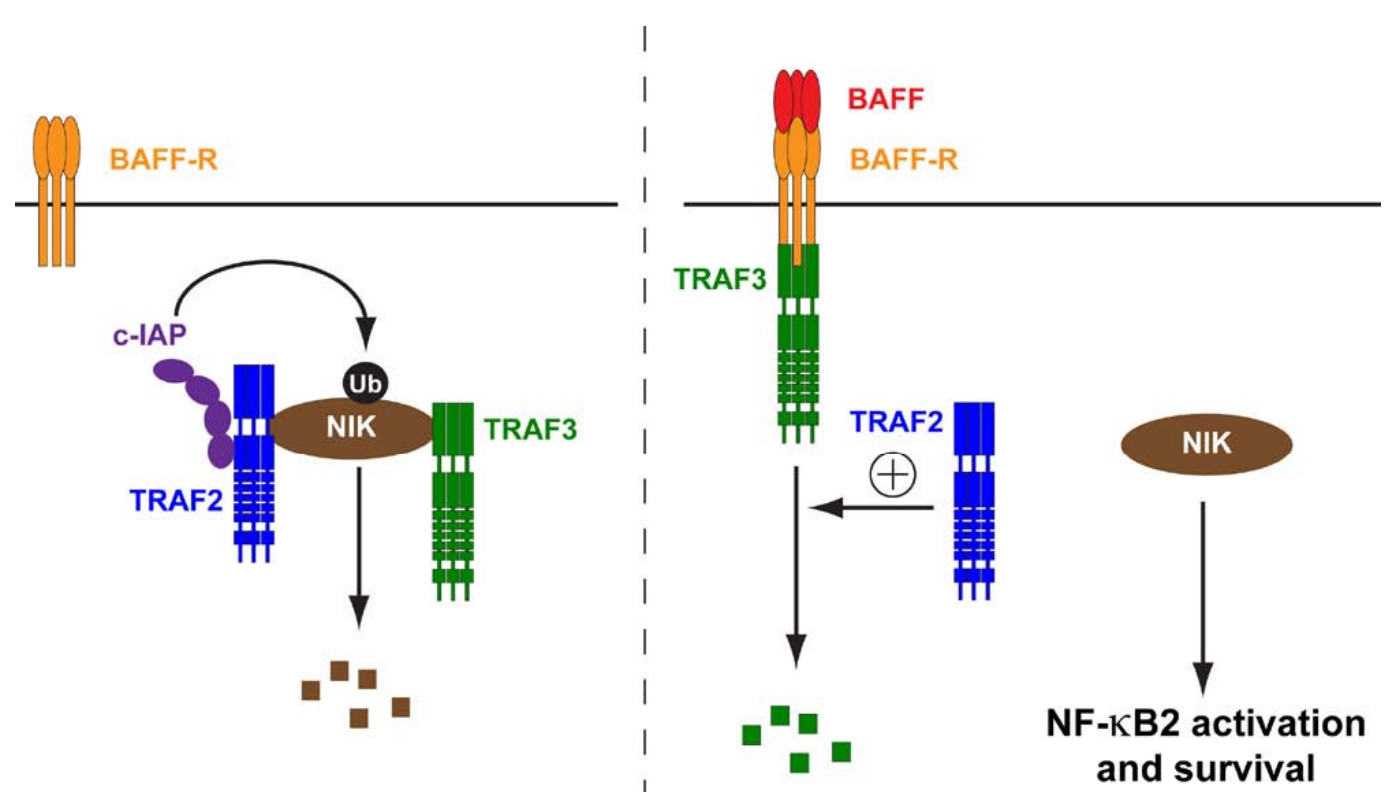

Mackay et al, Fig 2 


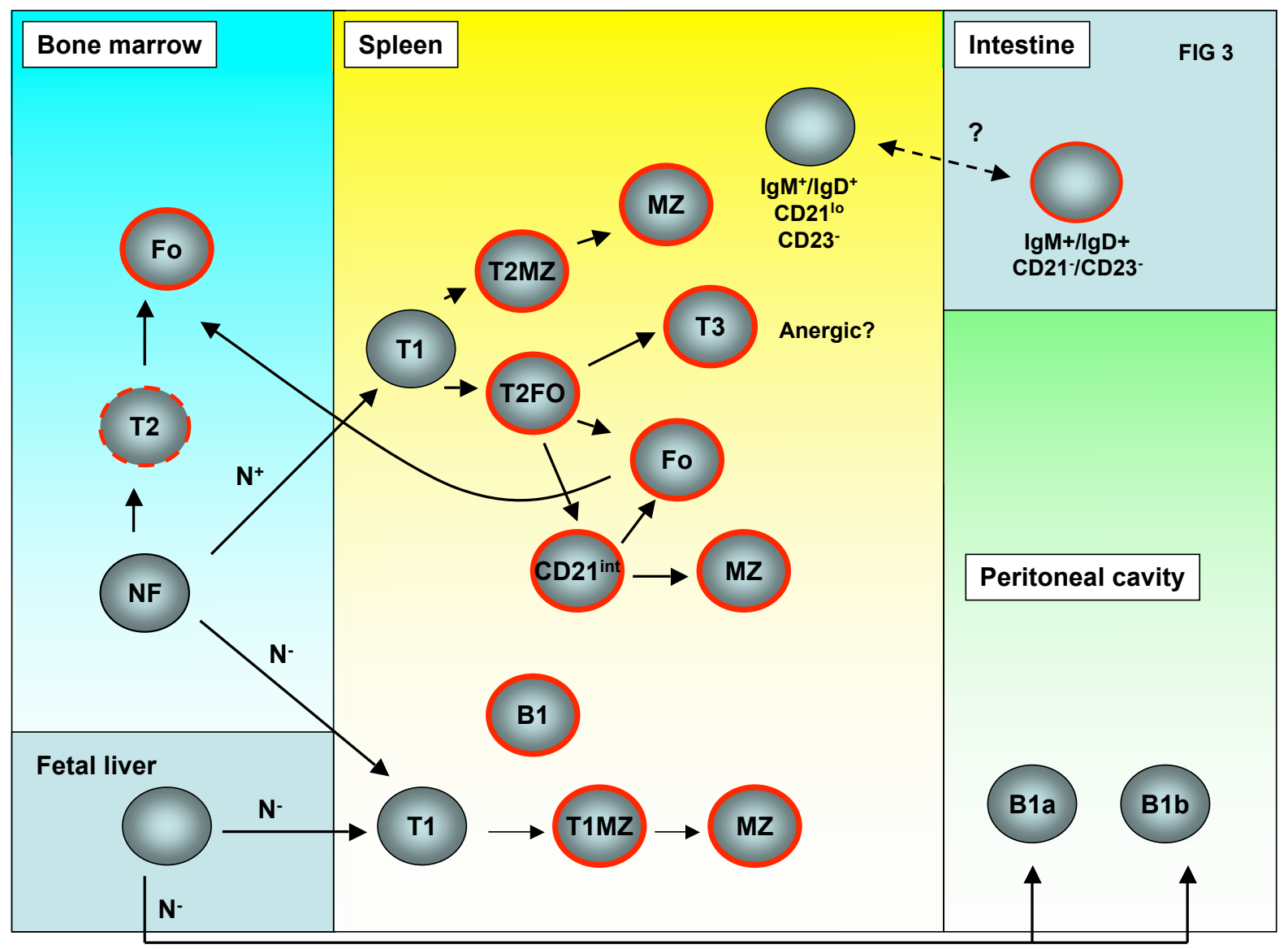




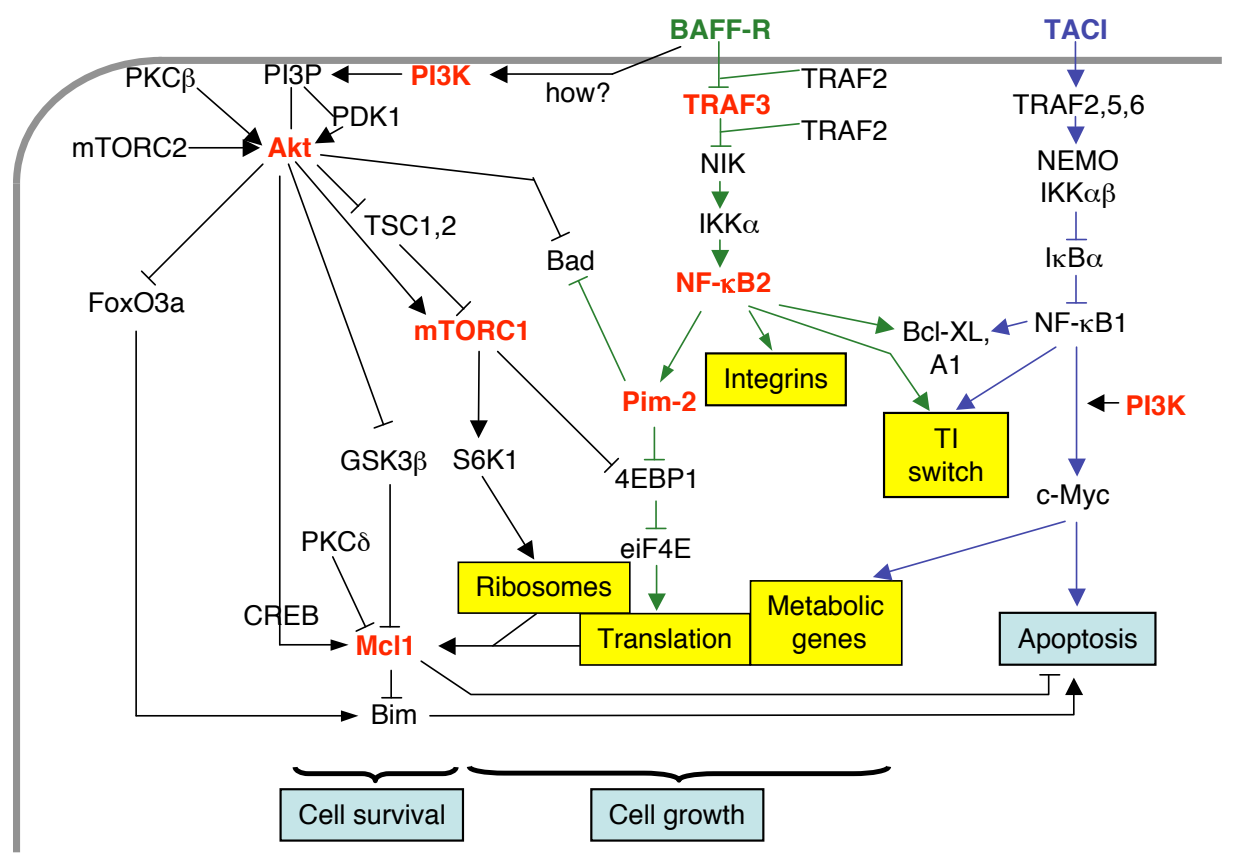

Mackay et al, Fig 4 


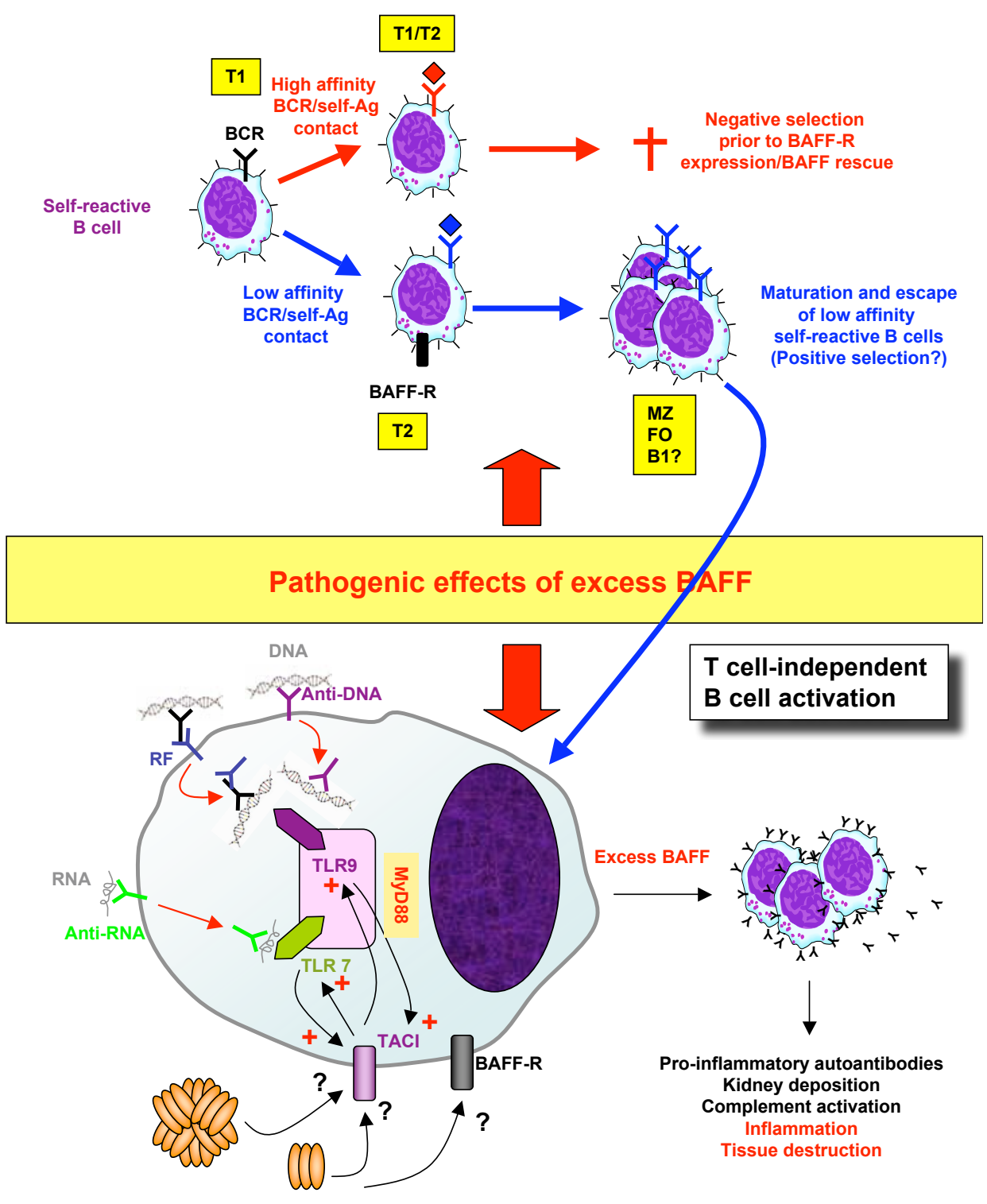

